\title{
Rückenmarksnahe Analgesien während der Geburt
}

Rückenmarksnahe Anästhesien werden heutzutage zur Analgesie und zur Erleichterung der Geburt je nach Klinik bei 10 bis $60 \%$ aller Geburten eingesetzt. In den meisten Fällen wird ein Katheter in den lumbalen Epiduralraum eingeführt (lumbale Epiduralanalgesie). Die Indikation für diese Methode wird vom Facharzt Gynäkologie und Geburtshilfe gestellt, die praktische Durchführung obliegt dem Facharzt für Anästhesiologie.

Bei der kaudalen Epiduralanästhesie (Kaudalanalgesie) erfolgt der Zugang zum periduralen Raum über den Hiatus sakralis. Kombiniert mit einer Allgemeinanästhesie wird diese Methode für kleine Eingriffe wie Leistenhernienoperationen usw. bei Kindern unter 6 Jahren routinemässig angewendet. Wie die untenstehende Stellungnahme der geburtshilflichen Anästhesisten der Universitätsfrauenkliniken Basel, Bern und Genf erläutert, wird hingegen der Einsatz der Kaudalanalgesie bei Gebärenden als obsolet beurteilt. Trotzdem wird diese Methode neuerdings von seiten einzelner Hebammen propagiert und auch selbständig praktiziert. Die Vorstände der Schweizerischen Gesellschaft für Gynäkologie und Geburtshilfe (SGGG) sowie der Schweizerischen Gesellschaft für Anästhesiologie und Reanimation (SGAR) distanzieren sich von dieser Praxis und unterstützen die Stellungnahme der Kollegen Schneider, Petersen-Felix und Kern.

Prof. Patrick Hohlfeld, Präsident SGGG

Prof. Franz Frei, Präsident SGAR

\section{Die geburtshilfliche Kaudalanalgesie: eine Standortbestimmung}

\author{
Markus Schneider ${ }^{a}$, Christian Kern ${ }^{b}$, Steen Petersen-Felix ${ }^{c}$
}

\footnotetext{
a Prof. Dr. med., Departement Anästhesie, Universitätsfrauenklinik Basel
}

b PD Dr, médecin adjoint, responsable de l'Unité d'anesthésiologie gynéco-obstétrique et ophtalmologique, HUG, Genève

c PD Dr. med., Leiter Anästhesie, Frauenklinik, Inselspital, Bern
Die moderne geburtshilfliche Schmerztherapie basiert auf verschiedenen rückenmarksnahen Anästhesietechniken, die bei vergleichbarem Wirkungsprofil ein unterschiedliches Risikopotential aufweisen. Die kontinuierliche lumbale Epiduralanalgesie (EA) gilt als Standardverfahren, die Option einer Dosierungskontrolle durch die Schwangere oder ihre Hebamme (Patienten kontrollierte Epiduralanalgesie; PCEA) erlaubt eine individualisierte rückenmarksnahe Schmerztherapie. Die kombinierte Spinal-Epiduralanalgesie (CSEA) erweitert die Indikationen der klassischen EA, auf ihr beruht das Konzept der sogenannten «Walking epidural». In diesem Umfeld hat die klassische Technik der kaudalen EA, kurz Kaudalanalgesie (KA), ihre ursprüngliche Bedeutung verloren, obwohl sie als Relikt aus der Vergangenheit überlebt hat.

Bei Erwachsenen ist die Durchführung einer KA wegen anatomischer Variationen des Hiatus sacralis technisch anspruchsvoll, auf die Risiken der Kontamination und Infektion bzw. der Verletzung des Rektums und des vorangehenden kindlichen Teils wird in der Literatur hingewiesen. Um in der Eröffnungsphase eine ausreichende Blockausbreitung bis zu den tiefen thorakalen
Segmenten (Th10) zu erzielen, müssen bei der KA ein grösseres Medikamentenvolumen als bei der lumbalen EA und eine entsprechend höhere Medikamentendosis injiziert werden. Ungenügende Analgesie, dosisabhängige motorische Blockade und frühzeitige Beckenbodenerschlaffung sind anatomisch begründet, weil die kaudal liegenden sakralen Segmente des Rückenmarks vor denjenigen des thorakolumbalen Übergangs blockiert werden. Der Gefässreichtum des kaudalen Epiduralraums erleichtert die Medikamentenresorption, erhöht das Risiko einer akzidentellen intravenösen Injektion und damit assoziierter zentral-nervöser toxischer Nebenwirkungen. Eine Beeinträchtigung der Muskelkraft wirkt sich nicht nur auf das Wohlbefinden der Mutter unter der Geburt aus, sondern gilt auch als Risikofaktor für eine assistierte Geburt (Zange, Vakuum) bzw. eine kindliche Fehleinstellung infolge früher Beckenbodenerschlaffung.

Im Gegensatz zur KA entsprechen die Wirkungsprofile der lumbalen EA und der CSEA den Anforderungen an eine optimale Analgesie unter der Geburt. Wegen ihres Nebenwirkungspotentials muss die KA in der Geburtshilfe hingegen als obsolet bezeichnet werden. 